\title{
STUDY OF SURFACE WAVE PROPAGATION IN A COAXIAL WAVEGUIDE WITH A PERIODIC SLOT ARRAY
}

\author{
D. Alesini ${ }^{* \S}$, C. Garganese ${ }^{\S}$, F. Iannazzo ${ }^{\S}$, M. Migliorati ${ }^{* \S}$, L. Palumbo*§ \\ * INFN - Laboratori Nazionali di Frascati, 00044 Frascati, Italy, \\ $\S$ University of Rome “LA SAPIENZA”, Dip. Energetica, 00161 Rome, Italy.
}

\begin{abstract}
In this paper we study the electromagnetic fields propagating in a coaxial waveguide with periodic slots in the inner conductor. We have performed a theoretic analysis by using the moment method, and have obtained a dispersion curve and an expression of the field components as a function of the geometrical parameters. The calculations show a slowing down of the phase velocity due to the slot array. We have then compared the predictions with the results of MAFIA simulation code and with measurements on a copper prototype.
\end{abstract}

\section{INTRODUCTION}

In order to study the coupling between the beam and the wake fields propagating in structures like the LHC liner [1], where pumping holes allow to create high vacuum in the beam pipe, we have analysed the electromagnetic (e. m.) fields of a coaxial waveguide with periodic slots in the inner conductor.

The theoretic study, described in the second section, has been performed with the moment method [2], and has allowed us to obtain a dispersion curve and an expression of the field components as a function of the geometrical parameters. The results show a slowing down of the wave phase velocity due to the slot array. We have applied the method to a case in which the slot dimensions produce an appreciable variation of phase velocity, and in the third section we have compared the results with those obtained with MAFIA simulation code [3]. Due to discrepancies between the two approaches, we have performed some measurements on a copper prototype, which, as shown in the fourth section, confirmed the MAFIA results. We end the paper with possible explanation of the discrepancies with the theoretic predictions.

\section{THE MOMENT METHOD}

The geometry of the structure is shown in Fig. 1. Let us consider a surface wave propagating on an infinitely long coaxial cable with the time dependence $e^{j \omega t}$. Each surface wave satisfies Maxwell's equations and all the boundary conditions. The source is located in the region $z=\infty$ and need not to be considered explicitly.

The slot spacing is $p$, the slot length is $l$ and the width is $w$. The inner conductor has radius $b$ while the outer conductor has radius $c$. The conductors are assumed to have

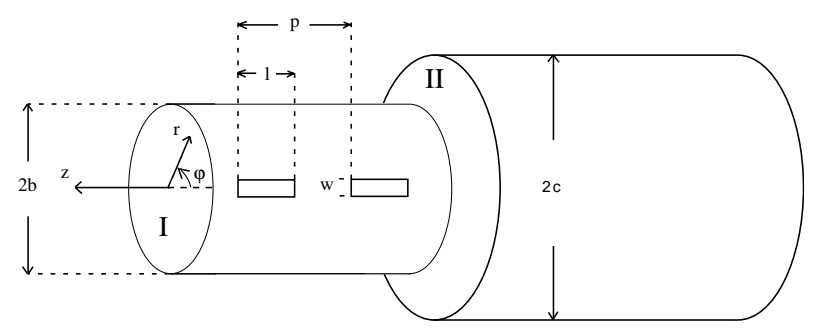

Figure 1: Geometry of the structure.

perfect conductivity and negligible thickness.

For each region I and II of Fig. 1, it is convenient to define electric and magnetic Hertz vectors $\Pi_{e}$ and $\Pi_{m}$, respectively, that have only $z$-components in terms of cylindrical coordinate system $(r, \varphi, z)$. These Hertz vectors must satisfy Floquet's theorem to include the effect of the periodic slot. Therefore we can write [4]

$$
\begin{aligned}
& \boldsymbol{\Pi}_{\boldsymbol{e}}^{(i)}=\sum_{m, n=-\infty}^{+\infty}\left[A_{m n}^{(i)} I_{n}+B_{m n}^{(i)} K_{n}\right] e^{j\left(n \varphi-\alpha_{m} z\right)} \boldsymbol{z}_{0} \\
& \boldsymbol{\Pi}_{\boldsymbol{m}}^{(i)}=\sum_{m, n=-\infty}^{+\infty}\left[C_{m n}^{(i)} I_{n}+D_{m n}^{(i)} K_{n}\right] e^{j\left(n \varphi-\alpha_{m} z\right)} \boldsymbol{z}_{0}
\end{aligned}
$$

where $i$ is the region I or II, $I_{n}$ and $K_{n}$ are the modified Bessel functions of argument $\left(\beta_{m} r\right)$, with $\beta_{m}=$ $\sqrt{\alpha_{m}^{2}-k^{2}}$ the radial propagation constant of the mth spatial harmonic, $\alpha_{m}=\alpha_{0}+2 \pi m / p$ the axial propagation constant, and $k$ the wave number. Since $K_{n}(x) \rightarrow \infty$ for $x \rightarrow 0$, it must be $B_{m n}^{(I)}=0$ and $D_{m n}^{(I)}=0$ to have a finite field everywhere.

In each region $i$ the e. m. fields can be expressed as

$$
\begin{aligned}
\boldsymbol{E} & =\nabla \nabla \cdot \boldsymbol{\Pi}_{\boldsymbol{e}}^{+} k^{2} \boldsymbol{\Pi}_{\boldsymbol{e}}-j \omega \mu_{0} \nabla \times \boldsymbol{\Pi}_{\boldsymbol{m}} \\
\boldsymbol{H} & =\nabla \nabla \cdot \boldsymbol{\Pi}_{\boldsymbol{m}}+k^{2} \boldsymbol{\Pi}_{\boldsymbol{m}}-j \omega \epsilon_{0} \nabla \times \boldsymbol{\Pi}_{\boldsymbol{e}}
\end{aligned}
$$

Our aim is to study the surface-waves $\left(\alpha_{m}^{2}>k^{2}\right)$ to obtain the constant propagation $\alpha_{0}$. In addition we determine the coefficients $A_{m n}^{(i)}, B_{m n}^{(i)}, C_{m n}^{(i)}, D_{m n}^{(i)}$ to study the field structure of the surface wave. To this end we require, as boundary conditions, that the tangential electric field in the two regions must be equal to the tangential electric field in the slots, which we call $E_{\varphi}^{(S)}$ and $E_{z}^{(S)}$. Since the fields are periodic functions in $\varphi$ and $z$, we can expand them in Fourier's series. By using the above boundary conditions, we get a linear system of equations which has the solu- 
tion [4]

$$
\begin{aligned}
A_{m n}^{(I)} & =-\frac{G_{m, n}}{\beta_{m}^{2} I_{n}\left(b_{m}\right)} \\
C_{m n}^{(I)} & =-j \frac{\left[\beta_{m}^{2} F_{m, n}+n \alpha_{m} G_{m, n}\right]}{\omega \mu_{0} \beta_{m}^{3} b I_{n}^{\prime}\left(b_{m}\right)} \\
A_{m n}^{(I I)} & =\frac{G_{m, n} K_{n}\left(c_{m}\right)}{\beta_{m}^{2}\left[K_{n}\left(b_{m}\right) I_{n}\left(c_{m}\right)-I_{n}\left(b_{m}\right) K_{n}\left(c_{m}\right)\right]} \\
B_{m n}^{(I I)} & =-\frac{G_{m, n} I_{n}\left(c_{m}\right)}{\beta_{m}^{2}\left[K_{n}\left(b_{m}\right) I_{n}\left(c_{m}\right)-I_{n}\left(b_{m}\right) K_{n}\left(c_{m}\right)\right]} \\
C_{m n}^{(I I)} & =-j \frac{K_{n}^{\prime}\left(c_{m}\right)\left[\beta_{m}^{2} F_{m, n}+n \alpha_{m} G_{m, n}\right]}{\omega \mu_{0} \beta_{m}^{3} b\left[K_{n}^{\prime}\left(c_{m}\right) I_{n}^{\prime}\left(b_{m}\right)-I_{n}^{\prime}\left(c_{m}\right) K_{n}^{\prime}\left(b_{m}\right)\right]} \\
D_{m n}^{(I I)} & =j \frac{I_{n}^{\prime}\left(c_{m}\right)\left[\beta_{m}^{2} F_{m, n}+n \alpha_{m} G_{m, n}\right]}{\omega \mu_{0} \beta_{m}^{3} b\left[K_{n}^{\prime}\left(c_{m}\right) I_{n}^{\prime}\left(b_{m}\right)-I_{n}^{\prime}\left(c_{m}\right) K_{n}^{\prime}\left(b_{m}\right)\right]}
\end{aligned}
$$

where $b_{m}=\beta_{m} b, c_{m}=\beta_{m} c$ and

$$
\begin{aligned}
F_{m, n} & =\frac{b}{2 \pi p} \int_{0}^{p} \int_{-\pi}^{\pi} E_{\varphi}^{(S)} e^{j \alpha_{0} z} e^{-j n \varphi} e^{\frac{j 2 \pi m}{p} z} d \varphi d z \\
G_{m, n} & =\frac{1}{2 \pi p} \int_{0}^{p} \int_{-\pi}^{\pi} E_{z}^{(S)} e^{j \alpha_{0} z} e^{-j n \varphi} e^{\frac{j 2 \pi m}{p} z} d \varphi d z
\end{aligned}
$$

To obtain the longitudinal propagation constant $\alpha_{0}$, we use the Galerkin's method [2]. We first expand the tangential components of the electric field in the slots as

$$
\begin{aligned}
& E_{\varphi}^{(S)}(\varphi, z)=\sum_{i=0}^{I-1} A_{i}^{\varphi} f_{i}(\varphi, z) \\
& E_{z}^{(S)}(\varphi, z)=\sum_{i=0}^{I-1} A_{i}^{z} g_{i}(\varphi, z)
\end{aligned}
$$

where $f_{i}(\varphi, z)$ and $g_{i}(\varphi, z)$ are the basis functions. Their choice depends on considerations about symmetries of the structure. For simplifying the calculations we have considered only the first term of the expansion $(I=1)$ and sinusoidal functions. As a result the fields on the slot become

$$
\begin{aligned}
& E_{\varphi}^{(S)}(\varphi, z)=A_{0}^{\varphi} \sin \left(\frac{\pi}{l} z\right) \sin \left(\frac{\pi b}{w} \varphi\right) e^{-j \alpha_{0} z} \\
& E_{z}^{(S)}(\varphi, z)=A_{0}^{z} \cos \left(\frac{\pi}{l} z\right) \cos \left(\frac{\pi b}{w} \varphi\right) e^{-j \alpha_{0} z}
\end{aligned}
$$

that can be seen as a first term of a Fourier series expansion.

The only parameters to be determined now are the coefficients $A_{0}^{\varphi}$ and $A_{0}^{z}$ and the constant of longitudinal propagation $\alpha_{0}$. To do that we use the conditions of matching the tangential components of the magnetic field between the two region through the slots. By using then the Galerkin's method we get a linear homogeneous system of equations [5]. The corresponding matrix determinant equal to zero gives the longitudinal propagation constant $\alpha_{0}$ as a function of frequency.

The phase velocity is related to $\alpha_{0}$ through the relation $v=\omega / \alpha_{0}$. In Fig. 2 we show the relative phase velocity

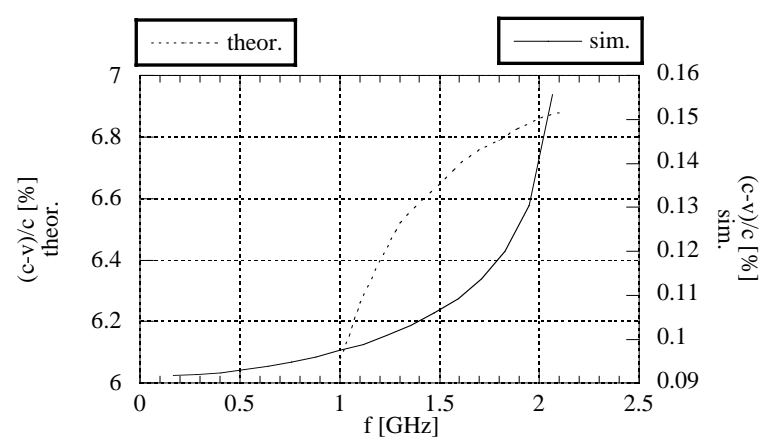

Figure 2: Relative phase velocity variation: theoretic and numerical results.

variation as a function of frequency for the case with dimensions: $a=19 \mathrm{~mm}, b=25 \mathrm{~mm}, w=8 \mathrm{~mm}, l=35$ $\mathrm{mm}$, and $p=70 \mathrm{~mm}$. The predicted variation can be rather consistent, of the order of $6-7 \%$ for a frequency of $1-2$ GHz.

\section{MAFIA SIMULATION RESULTS}

In order to check the results obtained in the previous section, we have evaluated the phase velocity of the slotted coaxial waveguide using the MAFIA e. m. simulation code. Due to the symmetry of the structure respect to the plane $\varphi=0$ (Fig. 3) and since we are interested in the TEM-like mode, we have simulated only half structure with a perfect magnetic plane boundary condition. Furthermore, since the structure is periodic, we have considered only one periodic cell with periodic boundary conditions on the planes $\mathrm{z}=0$ and $\mathrm{z}=\mathrm{p}$ and different phase advances between these two planes.

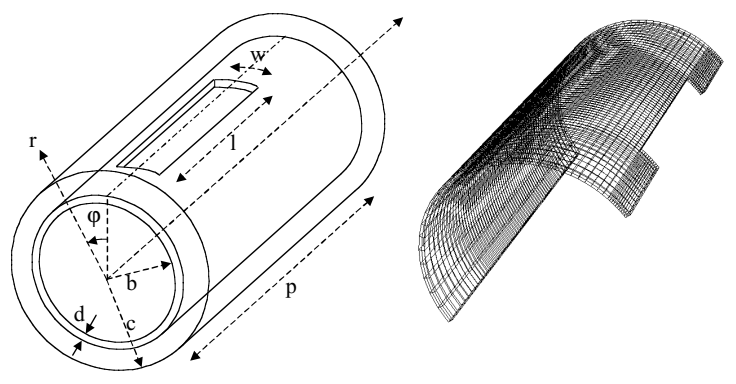

Figure 3: Sketch of the simulated structure and MAFIA meshgrid.

The considered structure is shown in Fig. 3. The characteristic dimensions are the same of the previous section with the exception of the inner conductor depth $d$ that is now $1 \mathrm{~mm}$. The total number of MAFIA meshpoints is 50000 .

The obtained relative phase velocity variation is plotted in Fig. 2 as a function of the frequency.

These results differ from the theoretic ones by a factor 


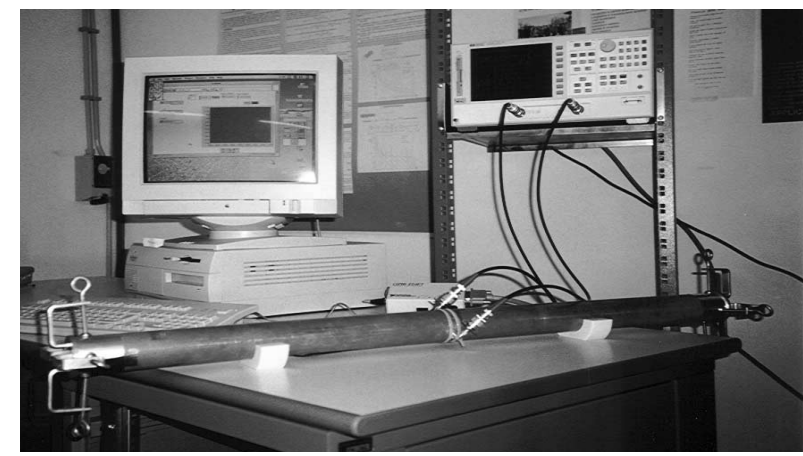

Figure 4: Measurement set-up.

bigger than 60 , and also the frequency dependence has not the same behavior. In order to check the validity of the simulations we have performed some measurements on a copper prototype.

\section{MEASUREMENTS}

To measure the slowing down of the wave in the region II, we have used the following method: we made a coaxial resonator out of the coaxial guide by applying a short circuit at the ends of the guide. The resonance frequencies of the resonator can be expressed as a function of the phase velocity of the wave in the region II

$$
f_{n}=\frac{v n}{2 L} \quad n=1,2, \ldots
$$

where $L=1043 \mathrm{~mm}$ is the length we have chosen for our resonator.

The slot array changes the phase velocity and therefore the resonance frequency. Let us consider $f_{n}^{(R E F)}$ and $f_{n}^{(D U T)}$ the resonance frequency of the nth mode for the simple and the slotted resonator respectively. From the measurement of the relative frequency shift, we obtain the slowing down of the phase velocity [6]

$$
\frac{f_{n}^{(R E F)}-f_{n}^{(D U T)}}{f_{n}^{(R E F)}}=\frac{c-v^{(D U T)}}{c}
$$

The measurement of the resonance frequency is obtained from the $\left|S_{21}\right|$ curve as a function of frequency. For this purpose we have used the Network Analyzer HP8753E. The complete set-up is shown in Fig. 4. All the measurements have been made for two structures of coaxial resonator: the resonator with no slot arrays (reference resonator), and the slotted coaxial resonator.

The geometrical parameters are the same as those used in the MAFIA simulations. The inner and outer cylinders of the resonators are made of copper; their ends have been closed with a couple of aluminum rings which create two short circuits at the terminal sections and assure the coaxiality of the entire structure. In order to excite and reveal the e. $\mathrm{m}$. field in the region II, we have used two probes in the middle of the resonator; for this reason we can study only modes corresponding to odd multiples of the fundamental resonance frequency. Furthermore we have fixed the aluminum rings with eight vices, to avoid eccentricities of the inner conductor and to stabilize the measurements, since the phase velocity variation predicted by MAFIA, and as a consequence the frequency shift, was very small.

We made $n=40$ repeated observations of the resonance frequency $f_{7}=7 f_{0}$. The average and the standard deviation are $\bar{f}_{7}^{(R E F)}=1007.48 \mathrm{MHz}, s^{(R E F)}\left(f_{k}\right)=0.20$ $\mathrm{MHz}$ for the reference resonator and $\bar{f}_{7}^{(D U T)}=1006.29$ $\mathrm{MHz}, s^{(D U T)}\left(f_{k}\right)=0.19 \mathrm{MHz}$ for the slotted resonator. The experimental standard deviation of the mean is $s^{(R E F)}(\bar{f}) \simeq 0.03 \mathrm{MHz} \simeq s^{(D U T)}(\bar{f})$.

The measured frequency shift $\bar{f}^{(R E F)}-\bar{f}^{(D U T)}$ is then $1.19 \mathrm{MHz}$. If we assume the combined standard uncertainty as a description of the uncertainty in the measurement, we get an error of $0.04 \mathrm{MHz}$.

These results give, for the frequency of $1007.48 \mathrm{MHz}$, a relative phase velocity of about $0.12 \%$ which confirms the MAFIA simulations. Our opinion is that the differences with the theory are due to the approximated tangential electric field components that we used to describe the field in the slots. In principle the moment method solution could be more accurate using more Fourier series terms for the field in the slots, but unfortunately this increases numerical and computational problems.

\section{CONCLUSIONS}

In this paper we have studied the characteristics of e.m. propagation in a coaxial waveguide with slots. Using the moment method we have obtained the dispersion diagram of the fundamental TEM-like mode that we have compared with the one obtained by MAFIA. The discrepancy in the estimation of the wave slowing down between the two approaches is probably due to the approximated tangential electric field components that we have used to describe the field in the slots. For a more accurate solution we should use more Fourier series terms for the field in the slots, but unfortunately this increases numerical and computational problems. We have also made e.m. measurements on a copper prototype that confirms the slowing down of the phase velocity predicted by MAFIA.

\section{REFERENCES}

[1] The LHC Study Group, CERN/AC/95-05(LHC), CERN, Geneva, October 1995.

[2] Roger F. Harrington, 'Field Computation by Moment Method', Macmillan, London 1968.

[3] CST, 'MAFIA: Manual', January 1999.

[4] Gi-Ho, Yun Seon-Taek, Kim and Han-Kiu Park, IEEE Trans. on Microwave Theory and Techniques. 46(3):269-279, 1998.

[5] F. Iannazzo, Dissertation, University of Rome, Rome, 2001.

[6] C. Garganese, Dissertation, University of Rome, Rome, 2001. 